(2) Open Access Full Text Article

PERSPECTIVES

\title{
Present-day challenges and future solutions in postoperative pain management: results from PainForum 2014
}

This article was published in the following Dove Press journal:

Journal of Pain Research

3 February 2016

Number of times this article has been viewed

\author{
Kristiina Kuusniemi' \\ Reino Pöyhiä ${ }^{2,3}$ \\ 'Department of Anaesthesiology, \\ Turku University, Turku, Finland; \\ ${ }^{2}$ Department of Anaesthesiology, \\ University of Helsinki, Helsinki, \\ Finland; ${ }^{3}$ Department of Palliative \\ Medicine and Oncology, University of \\ Turku, Turku, Finland
}

\begin{abstract}
This paper is a summary of presentations on postoperative pain control by the authors at the 2014 PainForum meeting in People's Republic of China. Postoperative pain is often untreated or undertreated and may lead to subsequent chronic pain syndromes. As more procedures migrate to the outpatient setting, postoperative pain control will become increasingly more challenging. Evidence-based guidelines for postoperative pain control recommend pain assessment using validated tools on a consistent basis. In this regard, consistency may be more important than the specific tool selected. Many hospitals have introduced a multidisciplinary acute pain service (APS), which has been associated with improved patient satisfaction and fewer adverse events. Patient education is an important component of postoperative pain control, which may be most effective when clinicians chose a multimodal approach, such as paracetamol (acetaminophen) and opioids. Opioids are a mainstay of postoperative pain control but require careful monitoring and management of side effects, such as nausea, vomiting, dizziness, and somnolence. Opioids may be administered using patient-controlled analgesia systems. Protocols for postoperative pain control can be very helpful to establish benchmarks for pain management and assure that clinicians adhere to evidence-based standards. The future of postoperative pain control around the world will likely involve more and better established APSs and greater communication between patients and clinicians about postoperative pain. The changes necessary to implement and move forward with APSs is not a single step but rather one of continuous improvement and ongoing change.
\end{abstract}

Keywords: pain control, postoperative pain, acute pain, pain assessment, analgesia

\section{Introduction}

This paper presents results from the 2014 PainForum meeting in People's Republic of China. The goal of the meeting was to describe the current state of postoperative pain management, identify challenges, and propose solutions for the future to allow for better and safe treatment of postoperative pain.

Even with our current knowledge and an armamentarium of analgesic agents, postoperative pain management has not changed much in the past two decades and much postoperative pain is not optimally treated. In a survey in the US dating back to $1995,57 \%$ of patients reported postsurgical pain as a concern prior to surgery and $80 \%$ reported moderate-to-severe pain after surgery. ${ }^{1}$ In a 2003 survey of 250 patients undergoing surgery, 33\% were concerned about pain during surgery and 59\% (the largest group) were concerned about pain following surgery. ${ }^{2}$ That fear is not unfounded: in that same survey, $82 \%$ of patients reported some degree of postsurgical pain, with $47 \%$ rating that pain moderate, $21 \%$ severe, and $18 \%$ extreme. ${ }^{2}$ A total of $30 \%-40 \%$
Correspondence: Kristiina Kuusniemi Department of Anaesthesiology, University of Turku, Fl-200 I 4 Turun Yliopisto, Finland

Email Kristiina.Kuusniemi@terveystalo. com 
of day surgery patients experience moderate-to-severe postoperative pain. ${ }^{3,4}$ Postoperative pain can delay discharge, may cause an unexpected hospital admission, ${ }^{5}$ and it can create pain, stress, and anxiety for patients and their family, possibly interfering with rehabilitation. ${ }^{4,6}$ Unplanned contacts between patient and physician following surgery are often the result of undermanaged pain; in Finland, 31\% of surgical patients made unplanned contact with clinical staff because of pain. ${ }^{7}$ In fact, pain is the main reason for unanticipated hospital admissions of day surgery patients $(36 \%){ }^{6}$

There are particularly important reasons for undertreated pain following ambulatory surgery, which is increasingly common. Outpatient procedures are more and more complex, and they are offered in the setting of many innovative new analgesic agents, techniques, and surgical tools. Ambulatory patients may have lower adherence than hospital inpatients who are under closer clinical supervision. In fact, left on their own, patients do not always use medications as prescribed, ${ }^{8}$ a phenomenon that has not changed much since 1979 when Sackett found typical adherence rates in patients with hypertension to be $\sim 50 \%$. $^{9}$

A variety of adverse outcomes have been associated with undertreated perioperative pain, including the possibility that chronic pain could result.

\section{The risks of chronic postsurgical pain}

The development of chronic pain syndromes following surgery is not rare and may be unappreciated by clinicians. The incidence of chronic postsurgical pain varies by surgery, but may be as high as $85 \%$ (amputations); patients who have undergone mastectomy face a $20 \%-50 \%$ incidence of chronic pain, who have undergone herniorrhaphy 5\%-35\%, who have undergone thoracotomy $30 \%-40 \%$, who have undergone breast surgery $20 \%-30 \%$, and who have undergone coronary artery bypass graft surgery $30 \%-50 \% .{ }^{10}$ The risk factors for developing chronic pain after surgery are several: preoperative pain, repeat surgery, prolonged surgery, younger age, severe postoperative pain, surgical approaches with a higher risk of nerve damage, chemotherapy or radiation, and some psychological or depressive symptoms. ${ }^{10}$ Some of these risk factors are beyond the control of the clinician, such as the degree of preoperative pain or the patient's age. The main risk factor under clinical control is the degree of postoperative pain the patient endures.

Numerous guidelines address the important subject of postoperative pain control. ${ }^{11-14}$ The American Society of Anesthesiology guidelines, updated in 2012, were created to facilitate safe and effective pain management in the perioperative setting, reduce adverse outcomes, maintain patient's function and well-being, and enhance the patient's quality of life in the perioperative period. ${ }^{15}$ A crucial first step in postoperative pain control according to the guidelines is regular, consistent pain assessment.

\section{Pain assessment and communication}

Pain assessment is recognized by virtually all of the pain guidelines as a crucial step in providing good pain management. The foundation of the pain assessment is the patient's self-report of pain, but most patients lack the ability to discuss pain in a clinically meaningful way. For that reason, a variety of tools have been introduced to help patients and clinicians "speak the same language" about the pain. Although clinicians may feel it is most natural to ask patients open-ended questions about their pain ("how are you feeling today?"), this is the least effective means of measuring pain because patients and clinicians are not calibrated to use terms the same way. Further, some patients avoid talking about pain because they think it might be a form of complaining, perceived as a sign of weakness, viewed by the doctors and nurses as bothersome, or that postsurgical pain is unavoidable. Pain assessments must be carried out frequently, ideally according to a schedule, because pain is dynamic and changes with the patient's condition, therapies, underlying disease, and other factors.

\section{Verbal descriptor scale}

The verbal descriptor scale (VDS) involves setting up five specific terms to describe pain; at the extremes are "no pain" and "worst pain possible". In addition to those two poles, the patient may select "mild", "moderate", or "severe" pain. Patients should be instructed to use only one of these five terms, and it is not uncommon in practice for patients to try to seek middle ground ("mild to moderate" or "moderate to severe"). The VDS is easy to use, intuitively understandable, and provides valuable pain assessment feedback. A potential drawback of this scale occurs when patients have difficulty communicating, for instance, because of language barriers or cognitive disorders. Note that for reliability in a clinical setting, clinicians must use the same terms each time.

\section{Visual analog scale}

The visual analog scale (VAS) involves a straight line of $10 \mathrm{~cm}$ with tick marks, starting with 0 and indicating each centimeter; smaller lines may indicate millimeters. The patient 
is instructed that 0 indicates no pain and $10 \mathrm{~cm}$ indicates the worst pain possible. The patient is then asked to indicate on the line "how much" pain he or she is experiencing. As a general rule, pain $\geq 3 \mathrm{~cm}$ demands treatment; pain $\geq 6 \mathrm{~cm}$ should be considered severe.

\section{Numerical rating scale}

The numerical rating scale works on a similar principle as the VAS, but instead of pinpointing the pain level on a straight line, the patient merely selects the number from 0 to 10 to describe his or her pain.

\section{Other pain assessment tools}

All of these pain assessment tools are easy to deploy and interpret. Other pain scales exist as well, such as the Red Wedge Scale and the Box Scale (a scale made up of 21 numbers in boxes). Some tools rely on photographs of faces or smiley/frowning cartoons to help patients describe their pain. ${ }^{16}$ These assessment tools may be particularly advantageous with pediatric patients or patients who do not speak the local language. A clinical practice should use only one assessment tool and use it consistently so that pain assessment results can be meaningfully compared and evaluated. In a study of 140 surgical patients with hip fracture or lower limb trauma, for elderly patients, the VDS and Red Wedge were the most applicable assessment tools, while the VAS was least reliable although still provided acceptable results. ${ }^{17}$

The use of one particular pain assessment tool over others is largely a matter of clinical or clinician preference. Optimal results require consistent use of one particular pain assessment tool in a clinical setting; however, it is less important which particular tool is used than that one tool be selected and used consistently.

Despite the wealth of pain assessment tools available, clinicians must often treat patients who are completely unable to communicate. In this instance, behavioral observations can be helpful. Distorted postures, grimacing, grunting, reluctance to move, muscle tension, agitation, difficulty breathing deeply, and problems sleeping may all indicate pain. ${ }^{18}$ When treating critically ill or uncommunicative patients, clinicians should consider assessing and comparing pain both during rest and with movement; however, behaviors that can suggest pain may also be indicators of potential surgical complications.

For practical reasons, postsurgical pain assessment carried out in the hospital must be brief and straightforward. When pain is identified, it should be promptly treated. This may require revamping institutional and procedural policies. Patients and clinical staff should be trained as to why prompt analgesic intervention is so important. Finally, hospitals and surgery centers should consider making it a criterion for discharge that the patient's pain is adequately controlled.

\section{The role of the acute pain service}

In the UK, hospitals have introduced a multidisciplinary acute pain service (APS) to manage postoperative pain, develop appropriate guidelines and training materials, conduct research into acute pain, and audit hospitals. ${ }^{19}$ The APS paradigm, which has been introduced in other parts of the world as well, holds promise in improving postoperative pain care and may help identify some of the risk factors for chronic postsurgical pain. Among these risks are patient factors (preoperative pain, repeat surgery, psychological vulnerability, preoperative anxiety, female sex, younger age, being on worker's compensation, etc), intraoperative factors (surgical approach with likelihood of nerve damage), and postoperative factors (radiation, chemotherapy, depression, psychological vulnerability, and moderate-to-severe acute pain). ${ }^{20}$

The benefits of APS include decreases in pain intensity levels among patients, improved patient satisfaction, and fewer adverse events associated with pain therapy. ${ }^{21}$ APS can better identify patients at risk for developing chronic postoperative pain and thus treating physicians can deploy more targeted interventions. Further, APS team members can provide education, training, and assurance that guidelines and evidence-based pain control are implemented.

\section{The importance of patient education}

Patients, even highly educated patients, may not know what to expect following surgery. To that end, it is helpful if the clinical team discusses pain expectations with the patient in advance, specifically, whether the patient should anticipate pain following surgery, how long the pain might be expected to last, how intense the pain might be, and what kind of pain treatments are available. A crucial step in surgical patient education is a frank discussion of the risks of untreated postsurgical pain with respect to development of chronic pain and delays in rehabilitation and recovery. Patients should also be informed about analgesic agents and their risks and benefits and encouraged to ask questions. It is not unusual to find that many patients underestimate or overestimate the potential risks of opioid analgesics.

Not all pain therapy has to be pharmacological. Where appropriate, patients should be told about nonpharmacological pain control options that can be very helpful, such as cold or heat therapy, massage, physical therapy, and they may also 
be urged to develop their own strategies to distract themselves from pain, for example, by listening to music or watching TV. When special techniques, such as an epidural injection or wound infiltrations, are used or new equipment such as patient-controlled analgesia (PCA) is introduced, patients should be instructed about their purpose and how they work. Patients are likely to be more compliant and better satisfied with their care when they understand their treatment. In a study of 322 surgical patients, a simple illustrated booklet about pain distributed to patients prior to surgery improved patient knowledge about the procedure and served to better standardize communication between patients and clinicians about pain and anesthesia. ${ }^{22}$

\section{Multimodal postoperative analgesia}

Postoperative pain is the likely outcome after surgery; for that reason, proactive approaches to pain control are appropriate for all surgical patients. This may include so-called preemptive analgesia, the preoperative administration of analgesic so that it is active during surgery and can reduce nociceptive transmissions (which may, in turn, decrease postoperative pain), and preventive analgesia to help block central sensitization by blocking neural transmission of noxious stimuli. Preoperatively, this might mean such treatments as nonsteroidal anti-inflammatory drugs (NSAIDs; eg, ketorolac), anxiolytics (eg, midazolam), and anticonvulsants (such as gabapentin), but data remain equivocal. ${ }^{23}$ Since postsurgical pain tends to be multimechanistic (for instance, having a nociceptive as well as neuropathic component), effective pain control involves multimodal therapy.

The term "multimodal analgesia" was coined by Kehlet to describe the combined use of different analgesics and/ or analgesic techniques to improve pain control. ${ }^{24}$ When analgesic agents with complementary mechanisms of action are utilized, there may be an additive benefit (the benefit is the result of the sum of the parts) or even a synergistic benefit (the benefit is greater than the sum of the parts). ${ }^{25}$ Furthermore, multimodal analgesia may reduce the adverse effects that would have occurred with monotherapy. Early and aggressive pain control may be able to slow the acute neuroplastic responses following surgery (leading to central sensitization). ${ }^{26,27}$

Multimodal analgesic combinations may involve nonopioid agents, opioids, or both. NSAIDs and acetaminophen have been shown to be effective as monotherapy against postsurgical pain ${ }^{28-30}$ and may be more effective in combination (NSAID/acetaminophen). ${ }^{31}$ Intravenous (IV) acetaminophen for postoperative pain control appears to be not only effective but also well tolerated. ${ }^{29}$ NSAIDs are effective against postoperative pain, but their effects may contraindicate their widespread use. For example, NSAIDs may interfere with platelet function, ${ }^{32,33}$ may be contraindicated in patients with compromised renal function, ${ }^{34}$ may cause or exacerbate gastrointestinal disorders, ${ }^{35,36}$ may promote bronchospasm in asthmatics sensitive to aspirin and/or certain NSAIDs, ${ }^{37}$ may interfere with bone healing, ${ }^{38}$ and may carry with them cardiovascular risks. ${ }^{39-41}$ NSAIDs may also interfere with the platelet effect of low-dose aspirin. ${ }^{42}$ However, nonopioid analgesics are appropriate and effective for control of mild to moderate pain.

For severe acute pain, the most commonly used medication in Finland remains oxycodone, which has become a cornerstone of postoperative pain control there. ${ }^{43,44}$ Oxycodone is available in IV, intramuscular, subcutaneous, intranasal, and per oral (PO) formulations. Oxycodone is well absorbed after PO dosing, with a bioavailability of around $60 \%-87 \%{ }^{45,46}$ Oxycodone intranasal has a mean bioavailability of $46 \% .{ }^{47}$ Oxycodone doses and routes of administration reported from Finnish hospitals are summarized in Table $1 .^{43}$

The safe and effective use of any opioid agent involves titrating the drug until the patient achieves analgesia as determined by the patient's own self-report. Patients who ask for more opioid usually have inadequate analgesia or are going too long between doses (so-called "analgesic gaps" are occurring) or the clinical team may not be paying adequate attention to the patient and his needs. ${ }^{48}$

\section{Considerations on the use of opioids for postoperative pain control}

$\mathrm{Mu}$-opioid receptor agonists are associated with certain well-known adverse events, which can in some instances limit treatment. Some can be antagonized with the nonselective opioid receptor agonist naloxone. Among these opioid-associated side effects are respiratory depression (potentially life threatening), difficulty breathing, nausea, vomiting, postoperative nausea and vomiting, sedation, somnolence, constipation, urinary retention, and itching. ${ }^{49}$ Many opioid-associated side effects can be managed, for example, nausea and vomiting with antiemetics. The use of opioid analgesics in the postoperative setting is generally brief, and the short duration of opioid exposure may limit the frequency and/or severity of typical opioid-associated side effects.

PCA allows the patient to control a device that administers a programmed amount of IV opioid. Devices can be 
Table I Oxycodone routes of administration and doses used in Finnish hospitals

\begin{tabular}{llllll}
\hline $\begin{array}{l}\text { Patient } \\
\text { ages (years) }\end{array}$ & $\begin{array}{l}\text { IV bolus } \\
\text { (dosing interval } \\
\text { I0-15 minutes) }\end{array}$ & $\begin{array}{l}\text { IV PCA (oxycodone } \mathbf{~ m g / m L ,} \\
\text { droperidol } \mathbf{0 . 0 5} \mathbf{~ m g} / \mathbf{m L} ; \\
\text { lockout 6-10 minutes) }\end{array}$ & $\begin{array}{l}\text { TM (dosing interval } \\
\text { I5-30 minutes) }\end{array}$ & $\begin{array}{l}\text { IR tablet PO or liquid } \\
\text { PO (dosing interval } \\
\text { CR tablet }\end{array}$ \\
(BID)
\end{tabular}

Note: Copyright ( $\odot$ 2012. Taylor \& Francis Ltd. Kokki H, Kokki M, Sjovall S. Oxycodone for the treatment of postoperative pain. Expert Opinion on Pharmacotherapy. 20 12; I3(7): 1045-1058. Reprinted by permission of the publisher (Taylor \& Francis Ltd, http://www.tandfonline.com). ${ }^{43}$

Abbreviations: BID, twice a day; CR, controlled release; IR, Immediate release; IV, intravenous; PCA, patient-controlled analgesia; PO, per oral (by mouth); TM, transmucosal.

programmed to specific concentrations, bolus doses, background infusions, and lockout times to prevent overdose. When a patient is given PCA equipment for postoperative pain control, it is important to educate the patient both in terms of device operation and medication risks. PCA patients should be regularly monitored, their pain regularly assessed, and drug consumption noted. PCA oxycodone (or other opioid) should be discontinued when the patient is not taking boluses regularly and is able to take PO medication; PCA oxycodone can be readily converted to oxycodone PO doses.

Epidural analgesics may be necessary for certain types of high-risk patients and can offer superior pain control compared with other techniques when a local anesthetic is combined with an opioid. ${ }^{50} \mathrm{In}$ a study of 18 mastectomy patients with patient-controlled epidural analgesia had significantly lower pain scores and significantly shorter hospital stays ( 25 hours) than similar patients who had PCA ${ }^{51}$ Other comparative studies have had equivocal results. ${ }^{52}$

Another technique for postoperative analgesia involves the localized infiltration of the deep tissue layers with a large volume of anesthetic solution and additive; the patient also has access to PCA opioids. The goal behind local infiltration is the theory that early, aggressive pain relief localized to the injury can prevent central sensitization. Local infiltration does not expose patients to the risk of epidural hematoma or motor block, and there is no risk of urinary retention. In a study of local infiltration analgesia for patients with knee and hip surgery, 325 patients were treated with the protocol of systematic infiltration of the tissue with ropivacaine, ketorolac, and adrenaline. Most patients did not require rescue morphine analgesia and most could walk with assistance within 5-6 hours following surgery; 71\% were discharged after one night in the hospital..$^{53}$

The types of infiltration solution vary. The literature has described levobupivacaine + adrenaline + ketorolac; ${ }^{54}$ other infiltrates might include NSAIDs, acetaminophen, and anticonvulsants. The patient should have access to rescue medication, as needed, such as PCA IV oxycodone or oxycodone PO (5-10 mg).

Regional anesthesia requires the clinician to put the right dose of the right drug in the right place. While there is not a body of evidence in the literature in support of ultrasound-guided regional blocks, it seems intuitively understandable that the correct use of ultrasound imaging would increase regional anesthesia safety. ${ }^{55}$ Regional anesthesia may be supplemented with certain adjuvant agents, such as adrenaline (to promote vasoconstriction) and clonidine (to help prolong analgesia by affecting A- $\alpha$ and C-fibers). ${ }^{56,57}$

\section{Best practices for postoperative pain control}

While some public health officials have argued that pain control is a fundamental human right, ${ }^{58,59}$ there are practical, clinical reasons for effectively controlling postoperative pain. ${ }^{60}$ First, there is growing evidence - along with the intuitive sense of most clinicians - that effective analgesia facilitates ambulation and rehabilitation.

The Royal College of Anesthetists has set forth best practice standards for postoperative pain control. ${ }^{61}$ For all patients for all surgeries, pain should be $<4$ on an 11-point VAS within 30 minutes in the recovery room. Patients should also be pain free at rest. Before patients are discharged, they should be given regular and breakthrough analgesic agents along with dosing instructions and, if necessary, antiemetic therapy. This is more complicated for patients at risk for cardiorespiratory morbidity; they may require more dynamic analgesia to adjust to their condition. If analgesia fails, this failure should be detected within no $>2$ hours and managed appropriately to restore analgesic relief.

While these principles seem reasonable and laudable, the challenge for busy real-world clinics is finding practical and efficient ways to implement best practices. That can be done 
by establishing pain protocols, using multimodal analgesic regimens, and setting up APS.

\section{Establishing protocols for postoperative pain}

A written protocol for best practices for postoperative pain should be developed. This should encompass certain established benchmarks: patients should be educated about the risk, benefits, and dosing instructions of their medicines and prescribed breakthrough pain medication (where appropriate) and antiemetic agents, if required. Patients should have a pain score on an 11-point scale of $<4$ within 30 minutes in the recovery room after surgery, with the goal that in recovery room, the patient can be pain free while at rest. This means pain should be assessed regularly using an established tool, which, in turn, means that the entire clinical team must be trained in the use of such tools. Postoperative analgesia should generally involve multimodal analgesia and may include IV or epidural PCA systems. The protocol should also allow for adjustments to the regimen in patients who are at risk for cardiorespiratory morbidity. Shortfalls, gaps, or failures of the analgesic protocol should be promptly detected and rectified, in no $>2$ hours. Further, the protocol set forth by a hospital should be subject to periodic audit.
A number of guidelines relating to postoperative pain control have been written in the past decades. A search in PubMed for "postoperative pain guidelines 2014" yielded 94 results from that year alone. The American Society of Anesthesiologists (ASA) revised its 2004 comprehensive guidelines in 2012 (Table 2). ${ }^{15}$

The ASA guidelines are succinct, comprehensive, and scientifically sound; they are based on the fundamental recognition that pain relief is important and that current practice must be continually reviewed and updated..$^{15}$ This leads to the question as to whether international postoperative pain relief guidelines could be formulated, based on the ASA guidelines and other international publications, such as work by $\mathrm{Wu}$ and Raja. ${ }^{62}$ Some drawbacks exist to the creation of global protocols. First, clinician education and institutional capacity vary, sometimes markedly, in different parts of the world. Second, the resources of health care systems vary by geography. Finally, patient populations as well as patient attitudes and expectations can vary in different parts of the world. For example, some cultures harbor profound aversions to opioids in any context; other cultures may accept pain stoically and consider it a sign of weakness to take pain relievers. None of these need to become obstacles, but they

Table 2 A brief summary of ASA guidelines for acute perioperative pain management

\begin{tabular}{|c|c|}
\hline Area & Recommendations \\
\hline \multirow[t]{6}{*}{ Institutional policies and procedures } & Training and education of health care providers \\
\hline & Monitoring patient outcomes and documentation \\
\hline & Monitoring patient outcomes at institutional level \\
\hline & Round-the-clock availability of anesthesiologists for perioperative pain management based on \\
\hline & standardized institutional policies and procedures \\
\hline & Use of APS \\
\hline \multirow[t]{3}{*}{ Preoperative evaluation of the patient } & Preoperative assessment and individualized plan (based on type of surgery, anticipated pain levels, \\
\hline & underlying conditions, risk/benefit of various analgesics, and patient preferences) \\
\hline & Physical examination and patient history \\
\hline \multirow[t]{6}{*}{ Preoperative preparation of the patient } & Adjustments or continuations of any medications that should not be discontinued because of \\
\hline & possible withdrawal symptoms \\
\hline & Treatments to reduce preexisting pain \\
\hline & Treatment to relieve anxiety \\
\hline & Premedications as part of a multimodal pain management program \\
\hline & Patient and family/caregiver education, including behavioral pain control techniques \\
\hline \multirow[t]{6}{*}{ Perioperative pain management } & May include, but is not limited to, central regional opioid analgesia, PCA with systemic opioid \\
\hline & agents, and peripheral regional analgesia \\
\hline & The abovementioned modalities should be given preference over intramuscular opioids "as needed" \\
\hline & Selections must reflect the expertise of the anesthesiology team and the capacity for safe application \\
\hline & at the practice setting \\
\hline & Exercise caution with continuous infusion modalities \\
\hline \multirow[t]{5}{*}{ Multimodal pain management } & Whenever possible, multimodal analgesic techniques should be used, such as central regional \\
\hline & blockade with local anesthetics \\
\hline & Unless contraindicated, patients should receive round-the-clock NSAIDs, coxibs, or acetaminophen \\
\hline & All dosing should optimize efficacy while minimizing the risk of adverse events \\
\hline & The choice of medication must be individualized (agent, dose, route, and duration of therapy) \\
\hline
\end{tabular}

Note: There are varying levels of evidence for these steps.

Abbreviations: ASA, American Society of Anesthesiologists; APS, acute pain service; NSAIDs, nonsteroidal anti-inflammatory drugs; PCA, patient-controlled analgesia. 
do merit consideration in formulation of global protocols. To set up any sort of basic preoperative pain relief protocol, established practices around the world should be reviewed and participation by local surgeons, anesthesiologists, and pain specialists is necessary. Furthermore, it must also be considered if there are any national differences in terms of who is in charge of postoperative pain control and how it is regulated.

The PAIN-OUT research project was set up to develop evidence-based approaches to improve postsurgical pain control by working with 14 collaborating hospitals in 13 nations. It achieved its proof-of-concept by ascertaining that it could obtain reasonably complete and good-quality data (only $2 \%$ of process and $0.06 \%$ of patient-reported outcome data were missing). ${ }^{63}$

The role of evidence in establishing sound postoperative pain protocols cannot be overstated. For example, epidural analgesia for postoperative pain was once considered a "gold standard" but that standard could not be supported by new evidence. ${ }^{64}$ It had previously been thought (based on "old evidence") that postoperative epidural analgesia decreased 30-day postoperative mortality, pneumonia, and deep vein thrombosis and shortened intensive care unit and hospital length of stay. However, scrutiny of this evidence has determined that some of those studies were flawed, that epidural analgesia did not have better mortality than systemic opioids, and that changes in surgical techniques and rehabilitation have impacted lengths of stay. ${ }^{64}$ The failure rate of epidural analgesia must also be considered, which has been stated as $32 \%-50 \% .^{65,66}$ Thus, epidural analgesia should be evaluated for postoperative analgesia only in highly selected cases and for patients who are otherwise at high risk for other analgesic regimens.

Surgical techniques, surgical tools, surgical procedures, and surgical facilities have all undergone profound changes in the past years. Many procedures which once required open surgical techniques can be performed laparoscopically, the number of procedures and patients who are appropriate candidates for outpatient procedures continues to expand, and advanced surgical tools and innovations have further facilitated once-complex procedures. These changes have had and continue to make a profound impact on postsurgical pain.

Specific postoperative pain management protocols have demonstrated effectiveness (Table 3 ) and may reduce pain, improve patient satisfaction, shorten hospital stay, reduce infections, improve sleep quality, lower readmission rates, and reduce side effects. Many studies have evaluated hospital data prior to the implementation of a protocol and then after the protocol was applied, often demonstrating a marked improvement in specific variables with the protocols.

\section{Optimizing postoperative pain control}

Multimodal analgesia may help optimize postoperative analgesia. In a meta-analysis of 52 randomized clinical trials ( $n=4,893$ adults), multimodal analgesia in postoperative patients was associated with 15\%-55\% less opioid consumption, $10 \%-15 \%$ lower postoperative VAS pain scores, and $15 \%-25 \%$ fewer opioid-associated adverse events. ${ }^{72}$ However, certain adverse events went up, such as severe bleeding (from $0 \%$ to $1.7 \%$ and renal insufficiency from $0 \%$ to $1.4 \%$ but this increase was seen only in cardiac patients).

Oxycodone works well in a multimodal analgesic plan. It is versatile, available as an PO, IV, or combination product, and can be used in PCA systems. Oxycodone has been used safely and effectively for postoperative pain control following diverse types of surgery, including, but not limited to, facial surgery, ${ }^{73}$ breast surgery, ${ }^{74}$ back surgery, ${ }^{74}$ ENT procedures, ${ }^{75}$ abdominal surgery, ${ }^{76}$ ocular procedures, ${ }^{77}$ neurosurgery, ${ }^{78}$ and urological procedures. ${ }^{79}$

Opioids are a broad class of drugs currently available in many different agents, doses, formulations, and routes of administration. Broadly, opioids may be grouped as mu-opioid receptor agonists, such as morphine; partial agonists, such as buprenorphine; and agonist-antagonists, such as butorphanol and pentazocine. While various opioid agents present unique pharmacokinetics and pharmacodynamics, there are some broad considerations about some of the best-known agents. Morphine, long considered the "gold standard" of opioid analgesia, has a slow onset of action and can be associated with toxicity and tolerability issues. Pethidine has a high toxicity. Fentanyl is a strong opioid, but it has a short duration of action and must be closely monitored to avoid the risk of accumulation in the body. Sufentanil is lipophilic. We should consider oxycodone in a special light here, because its use in postsurgical pain control is increasing globally. ${ }^{44}$

Oxycodone is a versatile opioid analgesic, in that it has demonstrated safety and effectiveness in experimental nociceptive pain, ${ }^{80,81}$ postoperative pain,${ }^{82,83}$ visceral pain, ${ }^{84-86}$ bone pain (fractures) ${ }^{87}$ cancer pain, ${ }^{88,89}$ hyperalgesic syndromes, ${ }^{80}$ and neuropathic pain. ${ }^{90}$

In a study of ten healthy volunteers, tolerance to PO oxycodone ( $0 \mathrm{mg}$, $5 \mathrm{mg}$, and $20 \mathrm{mg}$ ) was compared over 5 days. No difference in analgesic effect occurred from day 1 to 


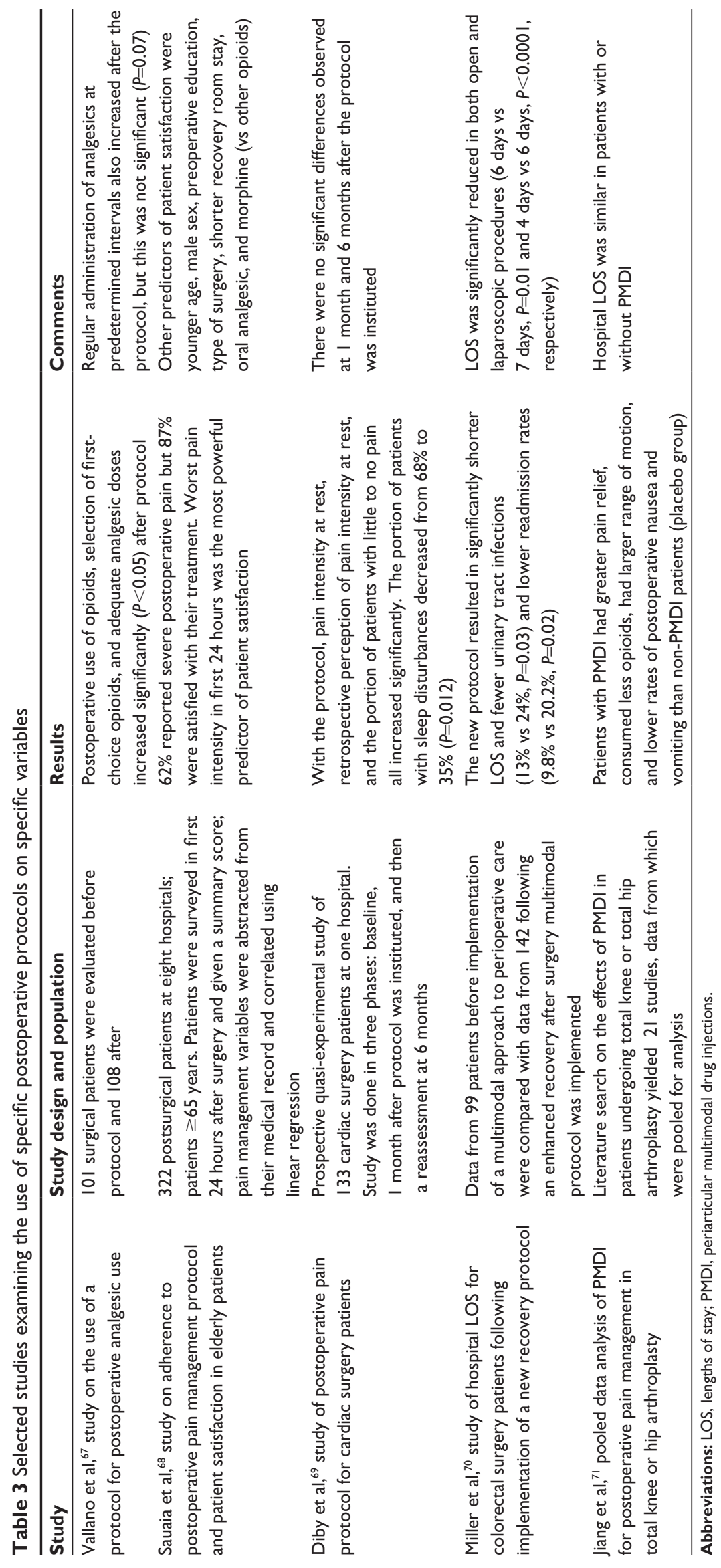


day 5, although tolerance did develop to some of the positive subjective effects of oxycodone in a subset of patients. ${ }^{91}$

Potential pharmacokinetic drug-drug interactions should be considered with oxycodone. Oyxcodone is metabolized via the cytochrome P2D6 and 3A4 substrates. Potential interactions may occur when the patient concurrently takes known 2D6 or 3A4 inhibitors or inducers. ${ }^{44,92,93}$ Genetic polymorphisms are known to affect other opioid agents, but only cytochrome $\mathrm{P}$ polymorphism affects oxycodone. ${ }^{94}$

In a double-blind study of 39 abdominal surgery patients, pain could be controlled over 2 hours with less oxycodone than morphine, and oxycodone was associated with fewer adverse events. ${ }^{76}$ In a study comparing oxycodone to fentanyl (median doses $15 \mathrm{mg}$ and $200 \mu \mathrm{g}$, respectively) in 78 laparoscopic cholecystectomy patients, pain intensity overall was significantly lower in the oxycodone group at 30 minutes, 60 minutes, and 90 minutes and at discharge from the postanesthesia care unit, with these differences achieving significance at 30 minutes and 60 minutes; both oxycodone IV and fentanyl IV had similar rapid onset of action ( $\sim 2$ minutes or 3 minutes after injection).$^{95}$ In a comparative study of oxycodone vs morphine over the first 24 hours after surgery, there was significantly more accumulated morphine detected than oxycodone $(22.0 \pm 13.1 \mathrm{mg}$ vs $13.3 \pm 10.4 \mathrm{mg}$, respectively, $P=0.006){ }^{96}$

Oxycodone may be easily switched from an IV route of administration to an $\mathrm{PO}$ product. ${ }^{97}$

\section{Clinical realities: past, present, and future}

The United States ${ }^{98}$ and Germany ${ }^{99}$ were the first nations to introduce the APS to perioperative care in 1985. By 1993, $34 \%$ of European hospitals surveyed in 17 countries had a formal APS program. ${ }^{19}$ By 1995, 42\% of the US hospitals had established an official APS. ${ }^{1,100}$ The APS model has introduced specialized pain control and new techniques (such as PCA and epidural analgesia) and has certain key overarching responsibilities: identifying training opportunities for the clinical team involved in postoperative pain, auditing existing procedures, and researching new and innovative methods of pain control. ${ }^{21}$ The structure of an APS may vary by institution to institution. Some are multidisciplinary organizations, others are led and run by nurses, and some are led and run by physicians, possibly in consultation with a pharmacist or physiotherapist. ${ }^{21}$

In a retrospective analysis based on 44 audits and four clinical trials ( $\mathrm{n}=84,097$ postoperative patients), an APS program significantly decreased pain scores ${ }^{21}$ and may reduce hospital length of stay for some surgeries. ${ }^{101}$ Data related to their cost-effectiveness are more limited, although one study reported a net savings of approximately US\$45 per patient with an APS, based on the cost of the program offset by shorter stays. ${ }^{102}$ In a large prospective study $(n=5,837)$, there were significantly fewer postoperative patients reporting "worst pain" in hospitals with an APS than in hospitals without $(P<0.00001) .{ }^{103}$ Postoperative patients in a hospital with APS reported significantly "less pain than expected after surgery" $(P<0.01) .{ }^{103}$ The incidence of lower respiratory tract infection decreased from $1.3 \%$ to $0.4 \%$ in a retrospective study once an APS was started $(P<0.01) .{ }^{104}$

The implementation of advanced analgesic techniques and a formal APS may lead to an increase in drug-related morbidity and adverse effects, although the relationship of APS and adverse events is not well studied. ${ }^{21}$

Despite what we know about multimodal analgesia, postoperative pain control, and the value of APS models, postoperative pain control is suboptimal around the world. In a questionnaire survey of 750 French surgical patients, $87 \%$ had postsurgical pain and $51 \%$ said it was severe to the worst pain imaginable. ${ }^{105}$ In the US, a phone survey $(n=250)$ found $82 \%$ of surgical patients had pain for 2 weeks following surgery and $21 \%$ said it was severe. ${ }^{2}$ The question arises: if we know what works, why does not the system work?

Postoperative pain control protocols exist and many are formally in place in hospitals and clinics, yet they may be unknown or incompletely known to clinicians and adherence is poor. With an increasingly strained and overburdened health care system, recovery room workloads may prevent careful adherence to protocols and, in some cases, there may not be a protocol in place for nurse-administered IV opioids. In some instances, clinical staff and hospital administration may not recognize the extreme importance of managing pain effectively. Poor protocol adherence and insufficient documentation may also occur.

It is the experience of the authors that change must be driven at an executive or administrative level of a facility. Once high-level advocates are found, clinicians can be mobilized to optimize care. Clinical change is not something that is "done"; it is a continuous process.

\section{Conclusion}

Pain management has come a long way, but much more needs to be done to provide optimal analgesia for patients dealing with postoperative or other acute pain syndromes. Pain must 
be assessed regularly, frequently, and with a validated instrument. Clinicians must learn more about pain and share this knowledge with their patients. While many guidelines exist, clinicians need to understand and implement these guidelines better. Hospitals can benefit greatly from APS teams who, in turn, can help develop more recommendations, guidelines, education activities, and research endeavors, to assist us in gathering the evidence we need to reliably fight pain. Finally, we need to advocate strongly for the use of multimodal analgesia as the standard of care for postoperative patients. When patients receive effective postoperative analgesia, it can reduce postoperative morbidity, enhance and accelerate recovery, shorten the hospital stay, and improve patient satisfaction. ${ }^{60}$ Considering the relatively low cost of analgesic agents, this type of treatment has a very favorable cost-to-benefit ratio.

\section{Acknowledgment}

Medical editing services were provided by LeQ Medical, Angleton, TX, USA, whose services were paid directly by Mundipharma.

\section{Disclosure}

The 2014 PainForum meeting in the People's Republic of China was sponsored by Mundipharma, which met all costs and paid expenses and honoraria to all presenters, including $\mathrm{KK}$ and RP. The authors were not compensated for this manuscript, and they have no other relevant disclosures.

\section{References}

1. Warfield CA, Kahn CH. Acute pain management. Programs in US hospitals and experiences and attitudes among US adults. Anesthesiology. 1995;83(5):1090-1094.

2. Apfelbaum JL, Chen C, Mehta SS, Gan TJ. Postoperative pain experience: results from a national survey suggest postoperative pain continues to be undermanaged. Anesth Analg. 2003;97(2):534-540, table of contents.

3. Beauregard L, Pomp A, Choiniere M. Severity and impact of pain after day-surgery. Can J Anaesth. 1998;45(4):304-311.

4. McGrath B, Elgendy H, Chung F, Kamming D, Curti B, King S. Thirty percent of patients have moderate to severe pain $24 \mathrm{hr}$ after ambulatory surgery: a survey of 5,703 patients. Can J Anaesth. 2004; 51(9):886-891.

5. Shaikh S, Chung F, Imarengiaye C, Yung D, Bernstein M. Pain, nausea, vomiting and ocular complications delay discharge following ambulatory microdiscectomy. Can J Anaesth. 2003;50(5):514-518.

6. Coley KC, Williams BA, DaPos SV, Chen C, Smith RB. Retrospective evaluation of unanticipated admissions and readmissions after same day surgery and associated costs. J Clin Anesth. 2002;14(5): 349-353.

7. Mattila K, Toivonen J, Janhunen L, Rosenberg PH, Hynynen M. Postdischarge symptoms after ambulatory surgery: first-week incidence, intensity, and risk factors. Anesth Analg. 2005;101(6):1643-1650.

8. Haynes RB, Ackloo E, Sahota N, McDonald HP, Yao X. Interventions for enhancing medication adherence. Cochrane Database Syst Rev. 2008;(2):CD000011.
9. Sackett DL. The hypertensive patient: 5. Compliance with therapy. Can Med Assoc J. 1979;121(3):259-261.

10. Kehlet $\mathrm{H}$, Jensen T, Woolf C. Persistsent postsurgical pain: risk factors and prevention. Lancet. 2006;367(9522):1618-1625.

11. Rawal N, De Andres J, Fischer H, et al. Postoperative Pain ManagementGood Clinical Practice. Sweden: European Society of Regional Anaesthesia and Pain Therapy; 2004.

12. HCANJ Best Practice Committee. Pain Management Guideline. Hamilton, NJ: Health Care Association of New Jersey; 2004.

13. Botti M, Kent B, Bucknall T, et al. Development of a Management Algorithm for Post-operative Pain (MAPP) after total knee and total hip replacement: study rationale and design. Implement Sci. 2014;9:110.

14. Nurses ASoP. An introduction to the ASPAN pain and comfort clinical guideline. J Perianesth Nurs. 2003;18(1):232-236.

15. American Society of Anesthesiologists Task Force on Acute Pain Management. Practice guidelines for acute pain management in the perioperative setting: an updated report by the American Society of Anesthesiologists Task Force on Acute Pain Management. Anesthesiology. 2012;116(2):248-273.

16. Wong DL, Baker CM. Pain in children: comparison of assessment scales. Pediatr Nurs. 1988;14(1):9-17.

17. Leino KA, Kuusniemi KS, Lertola KK, Olkkola KT. Comparison of four pain scales in patients with hip fracture or other lower limb trauma. Acta Anaesthesiol Scand. 2011;55(4):495-502.

18. Rose L, Haslam L, Dale C, Knechtel L, McGillion M. Behavioral pain assessment tool for critically ill adults unable to self-report pain. Am J Crit Care. 2013;22(3):246-254.

19. Rawal N, Allvin R. Acute pain services in Europe: a 17-nation survey of 105 hospitals. Eur J Anaesthesiol. 1998;15:354-363.

20. Corke P. Postoperative pain management. Austr Prescr. 2013;36(6): 202-205.

21. Werner MU, Soholm L, Rotboll-Nielsen P, Kehlet H. Does an acute pain service improve postoperative outcome? Anesth Analg. 2002;95(5):1361-1372, table of contents.

22. Cheung A, Finegan BA, Torok-Both C, Donnelly-Warner N, Lujic J. A patient information booklet about anesthesiology improves preoperative patient education. Can J Anaesth. 2007;54(5): 355-360.

23. Argoff CE. Recent management advances in acute postoperative pain. Pain Pract. 2014;14(5):477-487.

24. Kehlet H, Dahl JB. The value of "multimodal" or "balanced analgesia" in postoperative pain treatment. Anesth Analg. 1993;77(5): 1048-1056.

25. Tallarida RJ, Raffa RB. Testing for synergism over a range of fixed ratio drug combinations: replacing the isobologram. Life Sci. 1996;58(2): PL 23-28.

26. Elvir-Lazo OL, White PF. Postoperative pain management after ambulatory surgery: role of multimodal analgesia. Anesthesiol Clin. 2010;28(2):217-224.

27. Elvir-Lazo OL, White PF. The role of multimodal analgesia in pain management after ambulatory surgery. Curr Opin Anaesthesiol. 2010; 23(6):697-703.

28. Gorocs TS, Lambert M, Rinne T, Krekler M, Modell S. Efficacy and tolerability of ready-to-use intravenous paracetamol solution as monotherapy or as an adjunct analgesic therapy for postoperative pain in patients undergoing elective ambulatory surgery: open, prospective study. Int J Clin Pract. 2009;63(1):112-120.

29. Sinatra RS, Jahr JS, Reynolds L, et al. Intravenous acetaminophen for pain after major orthopedic surgery: an expanded analysis. Pain Pract. 2012;12(5):357-365.

30. Sinatra RS, Jahr JS, Reynolds LW, Viscusi ER, Groudine SB, Payen-Champenois C. Efficacy and safety of single and repeated administration of 1 gram intravenous acetaminophen injection (paracetamol) for pain management after major orthopedic surgery. Anesthesiology. 2005;102(4):822-831. 
31. Ong CK, Seymour RA, Lirk P, Merry AF. Combining paracetamol (acetaminophen) with nonsteroidal antiinflammatory drugs: a qualitative systematic review of analgesic efficacy for acute postoperative pain. Anesth Analg. 2010;110(4):1170-1179.

32. Serebruany VL, Malinin AI, Bhatt DL. Paradoxical rebound platelet activation after painkillers cessation: missing risk for vascular events? Am J Med. 2006;119(8):707.e11-707.e16.

33. Gladding PA, Webster MW, Farrell HB, Zeng IS, Park R, Ruijne N. The antiplatelet effect of six non-steroidal anti-inflammatory drugs and their pharmacodynamic interaction with aspirin in healthy volunteers. Am J Cardiol. 2008;101(7):1060-1063.

34. Brater DC. Anti-inflammatory agents and renal function. Semin Arthritis Rheum. 2002;32(3 Suppl 1):33-42.

35. Castellsague J, Riera-Guardia N, Calingaert B, et al; Safety of NonSteroidal Anti-Inflammatory Drugs (SOS) Project. Individual NSAIDs and upper gastrointestinal complications: a systematic review and meta-analysis of observational studies (the SOS project). Drug Saf. 2012;35(12):1127-1146.

36. Brooks J, Warburton R, Beales IL. Prevention of upper gastrointestinal haemorrhage: current controversies and clinical guidance. Ther $A d v$ Chronic Dis. 2013;4(5):206-222.

37. Morales DR, Lipworth BJ, Guthrie B, Jackson C, Donnan PT, Santiago VH. Safety risks for patients with aspirin-exacerbated respiratory disease after acute exposure to selective nonsteroidal antiinflammatory drugs and COX-2 inhibitors: Meta-analysis of controlled clinical trials. J Allergy Clin Immunol. 2014;134(1):40-45.

38. Jeffcoach DR, Sams VG, Lawson CM, et al; University of Tennessee Medical Center, Department of Surgery. Nonsteroidal anti-inflammatory drugs' impact on nonunion and infection rates in long-bone fractures. J Trauma Acute Care Surg. . 2014;76(3):779-783.

39. Farkouh ME, Greenberg JD, Jeger RV, et al. Cardiovascular outcomes in high risk patients with osteoarthritis treated with ibuprofen, naproxen or lumiracoxib. Ann Rheum Dis. 2007;66(6):764-770.

40. Vardeny O, Solomon SD. Cyclooxygenase-2 inhibitors, nonsteroidal anti-inflammatory drugs, and cardiovascular risk. Cardiol Clin. 2008;26(4):589-601.

41. Ong HT, Ong LM, Tan TE, Chean KY. Cardiovascular effects of common analgesics. Med J Malaysia. 2013;68(2):189-194.

42. Yokoyama H, Ito N, Soeda S, et al. Influence of non-steroidal antiinflammatory drugs on antiplatelet effect of aspirin. J Clin Pharm Ther. 2013;38(1):12-15.

43. Kokki H, Kokki M, Sjovall S. Oxycodone for the treatment of postoperative pain. Expert Opin Pharmacother. 2012;13(7):1045-1058.

44. Olkkola KT, Kontinen VK, Saari TI, Kalso EA. Does the pharmacology of oxycodone justify its increasing use as an analgesic? Trends Pharmacol Sci. 2013;34(4):206-214.

45. Poyhia R, Seppala T, Olkkola KT, Kalso E. The pharmacokinetics and metabolism of oxycodone after intramuscular and oral administration to healthy subjects. Br J Clin Pharmacol. 1992;33(6):617-621.

46. Leow KP, Smith MT, Williams B, Cramond T. Single-dose and steadystate pharmacokinetics and pharmacodynamics of oxycodone in patients with cancer. Clin Pharmacol Ther. 1992;52(5):487-495.

47. Takala A, Kaasalainen V, Seppala T, Kalso E, Olkkola KT. Pharmacokinetic comparison of intravenous and intranasal administration of oxycodone. Acta Anaesthesiol Scand. 1997;41(2):309-312.

48. McQuay HJ, Moore RA. Postoperative analgesia and vomiting, with special reference to day-case surgery: a systematic review. Health Technol Assess. 1998;2(12):1-236.

49. Smith H, McCleane G, Thompson G. Assessing and managing opioid side effects. In: Smith H, Portenoy R, editors. Opioid Therapy in the 21st Century. New York: Oxford University Press; 2013: 79-104.

50. Breivik H, Bang U, Jalonen J, Vigfusson G, Alahuhta S, Lagerkranser M. Nordic guidelines for neuraxial blocks in disturbed haemostasis from the Scandinavian Society of Anaesthesiology and Intensive Care Medicine. Acta Anaesthesiol Scand. 2010;54(1):16-41.
51. Correll DJ, Viscusi ER, Grunwald Z, Moore JH Jr. Epidural analgesia compared with intravenous morphine patient-controlled analgesia: postoperative outcome measures after mastectomy with immediate TRAM flap breast reconstruction. Reg Anesth Pain Med. 2001;26(5):444-449.

52. Correll DJ, Vlassakov KV, Kissin I. No evidence of real progress in treatment of acute pain, 1993-2012: scientometric analysis. J Pain Res. 2014;7:199-210

53. Kerr DR, Kohan L. Local infiltration analgesia: a technique for the control of acute postoperative pain following knee and hip surgery: a case study of 325 patients. Acta Orthop. 2008;79(2):174-183.

54. Rikalainen-Salmi R, Förster JG, Mäkelä K, et al. Local infiltration analgesia with levobupivacaine compared with intrathecal morphine in total hip arthroplasty patients. Acta Anaesthesiol Scand. 2012; 56(6):695-705.

55. Marhofer P, Willschke H, Kettner S. Current concepts and future trends in ultrasound-guided regional anesthesia. Curr Opin Anaesthesiol. 2010;23(5):632-636.

56. Murphy DB, McCartney CJ, Chan VW. Novel analgesic adjuncts for brachial plexus block: a systematic review. Anesth Analg. 2000; 90(5):1122-1128

57. Liu SS, Salinas FV. Continuous plexus and peripheral nerve blocks for postoperative analgesia. Anesth Analg. 2003;96(1):263-272.

58. Brennan F, Carr DB, Cousins M. Pain management: a fundamental human right. Anesth Analg. 2007;105(1):205-221.

59. International Pain Summit of The International Association for The Study of Pain. Declaration of Montreal: declaration that access to pain management is a fundamental human right. J Pain Palliat Care Pharmacother. 2011;25(1):29-31.

60. Kehlet H. Postoperative pain relief - what is the issue? Br J Anaesth. 1994;72(4):375-378.

61. The Royal College of Anaesthetists. Clinical Quality, Standards and Safety; 2014. Available from: http://www.rcoa.ac.uk/clinical-standardsquality. Accessed November 30, 2014.

62. Wu CL, Raja SN. Treatment of acute postoperative pain. Lancet. 2011;377(9784):2215-2225.

63. Zaslansky R, Chapman CR, Rothaug J, et al. Feasibility of international data collection and feedback on post-operative pain data: proof of concept. Eur J Pain. 2012;16(3):430-438.

64. Rawal N. Epidural technique for postoperative pain: gold standard no more? Reg Anesth Pain Med. 2012;37(3):310-317.

65. Low JA, Johnston N, Morris CB. Epidural analgesia: first do no harm [editorial]. Anaesthesia. 2008;63:1-3.

66. Ready L. Acute pain: lessons learned from 25,000 patients. Reg Anesth Pain Med. 1999;24:499-505.

67. Vallano A, Llinares J, Amau JM, Martorell M, Girona L, Laporte JR. Impact of analgesic drug-use guidelines for the management of postoperative pain: a drug utilization study. Int J Clin Pharmacol Ther. 2003; 41(4):165-170.

68. Sauaia A, Min SJ, Leber C, Erbacher K, Abrams F, Fink R. Postoperative pain management in elderly patients: correlation between adherence to treatment guidelines and patient satisfaction. J Am Geriatr Soc. 2005;53(2):274-282.

69. Diby M, Romand JA, Frick S, Heidegger CP, Walder B. Reducing pain in patients undergoing cardiac surgery after implementation of a quality improvement postoperative pain treatment program. J Crit Care. 2008;23(3):359-371.

70. Miller TE, Thacker JK, White WD, et al; Enhanced Recovery Study Group. Reduced length of hospital stay in colorectal surgery after implementation of an enhanced recovery protocol. Anesth Analg. 2014;118(5):1052-1061.

71. Jiang J, Teng Y, Fan Z, Khan MS, Cui Z, Xia Y. The efficacy of periarticular multimodal drug injection for postoperative pain management in total knee or hip arthroplasty. J Arthroplasty. 2013;28(10):1882-1887.

72. Abbey J, Piller N, De Bellis A, et al. The Abbey pain scale: a 1-minute numerical indicator for people with end-stage dementia. Int J Palliat Nurs. 2004;10(1):6-13. 
73. Silvasti M, Tarkkila P, Tuominen M, Svartling N, Rosenberg PH. Efficacy and side effects of tramadol versus oxycodone for patientcontrolled analgesia after maxillofacial surgery. Eur J Anaesthesiol. 1999;16(12):834-839.

74. Silvasti M, Rosenberg P, Seppala T, Svartling N, Pitkanen M. Comparison of analgesic efficacy of oxycodone and morphine in postoperative intravenous patient-controlled analgesia. Acta Anaesthesiol Scand. 1998;42(5):576-580.

75. Salonen A, Kokki H, Nuutinen J. Recovery after tonsillectomy in adults: a three-week follow-up study. Laryngoscope. 2002;112(1): 94-98.

76. Kalso E, Poyhia R, Onnela P, Linko K, Tigerstedt I, Tammisto T. Intravenous morphine and oxycodone for pain after abdominal surgery. Acta Anaesthesiol Scand. 1991;35(7):642-646.

77. Kaufmann J, Yesiloglu S, Patermann B, Krombach J, Kiencke P, Kampe S. Controlled-release oxycodone is better tolerated than intravenous tramadol/metamizol for postoperative analgesia after retinalsurgery. Curr Eye Res. 2004;28(4):271-275.

78. Rahimi SY, Vender JR, Macomson SD, French A, Smith JR, Alleyne CH Jr. Postoperative pain management after craniotomy: evaluation and cost analysis. Neurosurgery. 2006;59(4):852-857; discussion 857.

79. Pedersen KV, Olesen AE, Drewes AM, Osther PJ. Morphine versus oxycodone analgesia after percutaneous kidney stone surgery: A randomised double blinded study. Urolithiasis. 2013;41(5):423-430.

80. Olesen AE, Staahl C, Arendt-Nielsen L, Drewes AM. Different effects of morphine and oxycodone in experimentally evoked hyperalgesia: a human translational study. Br J Clin Pharmacol. 2010; 70(2):189-200.

81. Olesen AE, Upton R, Foster DJ, et al. A pharmacokinetic and pharmacodynamic study of oral oxycodone in a human experimental pain model of hyperalgesia. Clin Pharmacokinet. 2010;49(12):817-827.

82. Hwang BY, Kwon JY, Kim E, Lee DW, Kim TK, Kim HK. Oxycodone vs fentanyl patient-controlled analgesia after laparoscopic cholecystectomy. Int J Med Sci. 2014;11(7):658-662.

83. Raeder J. Opioids in the treatment of postoperative pain: old drugs with new options? Expert Opin Pharmacother. 2014;15(4):449-452.

84. Mercadante S, Tirelli W, David F, et al. Morphine versus oxycodone in pancreatic cancer pain: a randomized controlled study. Clin J Pain. 2010;26(9):794-797.

85. Liguori S, Gottardi M, Micheletto G, Bruno L. Pharmacological approach to chronic visceral pain. Focus on oxycodone controlled release: an open multicentric study. Eur Rev Med Pharmacol Sci. 2010; 14(3):185-190.

86. Staahl C, Dimcevski G, Andersen SD, et al. Differential effect of opioids in patients with chronic pancreatitis: an experimental pain study. Scand J Gastroenterol. 2007;42(3):383-390.

87. Marco CA, Plewa MC, Buderer N, Black C, Roberts A. Comparison of oxycodone and hydrocodone for the treatment of acute pain associated with fractures: a double-blind, randomized, controlled trial. Acad Emerg Med. 2005;12(4):282-288.

88. King SJ, Reid C, Forbes K, Hanks G. A systematic review of oxycodone in the management of cancer pain. Palliat Med. 2011;25(5):454-470.

89. Riley J, Branford R, Droney J, et al. Morphine or oxycodone for cancerrelated pain? A randomized, open-label, controlled trial. J Pain Symptom Manage. 2015;49(2):161-172.

Journal of Pain Research

\section{Publish your work in this journal}

The Journal of Pain Research is an international, peer-reviewed, open access, online journal that welcomes laboratory and clinical findings in the fields of pain research and the prevention and management of pain. Original research, reviews, symposium reports, hypothesis formation and commentaries are all considered for publication.

Submit your manuscript here: http://www.dovepress.com/journal-of-pain-research-journal
90. Nunez Olarte JM. Oxycodone and the challenge of neuropathic cancer pain: a review. Oncology. 2008;74(Suppl 1):83-90.

91. Cooper ZD, Sullivan MA, Vosburg SK, et al. Effects of repeated oxycodone administration on its analgesic and subjective effects in normal, healthy volunteers. Behav Pharmacol. 2012;23(3):271-279.

92. Liukas A, Kuusniemi K, Aantaa R, et al. Elimination of intravenous oxycodone in the elderly: a pharmacokinetic study in postoperative orthopaedic patients of different age groups. Drugs Aging. 2011; 28(1):41-50

93. Gronlund J, Saari TI, Hagelberg NM, Neuvonen PJ, Laine K, Olkkola KT. Effect of inhibition of cytochrome P450 enzymes 2D6 and 3A4 on the pharmacokinetics of intravenous oxycodone: a randomized, three-phase, crossover, placebo-controlled study. Clin Drug Investig. 2011;31(3):143-153.

94. Moriyama A, Nishizawa D, Kasai S, et al. Association between genetic polymorphisms of the beta1-adrenergic receptor and sensitivity to pain and fentanyl in patients undergoing painful cosmetic surgery. J Pharmacol Sci. 2013;121(1):48-57.

95. Koch S, Ahlburg P, Spangsberg N, Brock B, Tonnesen E, Nikolajsen L. Oxycodone vs fentanyl in the treatment of early post-operative pain after laparoscopic cholecystectomy: a randomised double-blind study. Acta Anaesthesiol Scand. 2008;52(6):845-850.

96. Lenz H, Sandvik L, Qvigstad E, Bjerkelund CE, Raeder J. A comparison of intravenous oxycodone and intravenous morphine in patientcontrolled postoperative analgesia after laparoscopic hysterectomy. Anesth Analg. 2009;109(4):1279-1283.

97. Kokki M, Välitalo P, Rasanen I, et al. Absorption of different oral dosage forms of oxycodone in the elderly: a cross-over clinical trial in patients undergoing cystoscopy. Eur J Clin Pharmacol. 2012; 68(10):1357-1363.

98. Petrakis J. Acute pain services in a community hospital. Clin J Pain. 1989;68:100-106.

99. Maier C, Kibbel K, Mercker S, Wulf H. Postoperative pain in therapy at general nursing stations: an analysis of eight years experience at an anesthesiological acute pain service. Anaesthetist. 1994; 43(6):385-397.

100. Ready LB. How many acute pain services are there in the United States, and who is managing patient-controlled analgesia? Anesthesiology. 1995;82(1):322.

101. Tsui SL, Law S, Fok M, et al. Postoperative analgesia reduces mortality and morbidity after esophagectomy. Am J Surg. 1997; 173(6):472-478.

102. Brodner G, Mertes N, Buerkle H, Marcus MA, Van Aken H. Acute pain management: analysis, implications and consequences after prospective experience with 6349 surgical patients. Eur J Anaesthesiol. 2000;17(9):566-575.

103. Miaskowski C, Crews J, Ready LB, Paul SM, Ginsberg B. Anesthesiabased pain services improve the quality of postoperative pain management. Pain. 1999;80(1-2):23-29.

104. Wheatley R, Madej T, Jackson I, Hunter DJ. The first year's experience of an acute pain service. Br J Anaesth. 1991;67:353-359.

105. Fletcher D, Fermanian C, Mardaye A, Aegerter P, Comité DouleurALR de la Sfar. A patient-based national survey on postoperative pain management in France reveals significant achievements and persistent challenges. Pain. 2008;137:441-451.

The manuscript management system is completely online and includes a very quick and fair peer-review system, which is all easy to use. Visit http://www.dovepress.com/testimonials.php to read real quotes from published authors. 